\title{
Long Wavelength Limit of the Current Convective Instability
}

\author{
J. D. HuBA \\ Geophysical and Plasma Dynamics Branch, Plasma Physics Division, Naval Research Laboratory
}

A linear theory is presented of the current convective instability in the long wavelength limit, i.e., $k L \ll 1$ where $k$ is the wave number and $L$ is the scale length of the density inhomogeneity. A relatively simple dispersion equation is derived that describes the modes in this limit. Analytical solutions are presented in both the collisional $\left(v_{i n} \gg \omega\right)$ and inertial $\left(v_{i n} \ll \omega\right)$ limits where $\omega$ is the wave frequency and $v_{i n}$ is the ion-neutral collision frequency. It is shown that the growth rate scales as $k$ in the collisional limit and as $k^{2 / 3}$ in the inertial limit. The analytical solutions are compared to exact numerical solutions, and very good agreement is found. Applications to the auroral ionosphere are discussed.

\section{INTRODUCTION}

The current convective instability has recently been suggested as a mechanism to generate density irregularities in the auroral ionosphere [Ossakow and Chaturvedi, 1979; Vickrey et al., 1980; Chaturvedi and Ossakow, 1981; Keskinen and Ossakow, 1982, 1983]. These irregularities can cause the scintillation phenomena observed by the Defense Nuclear Agency (DNA) wideband satellite during periods of diffuse aurora [Fremouw et al., 1977; Rino et al., 1978] and will be an important area of study of the DNA HILAT satellite mission [Fremouw et al., 1983]. The current convective instability can become unstable in a plasma that supports a density gradient (perpendicular to the ambient magnetic field) and a current that flows parallel to the ambient B field; a situation which can occur in the diffuse auroral zone.

The instability was initially studied to understand plasma disturbances in laboratory experiments [Lehnert, 1958; Hoh and Lehnert, 1960; Kadomtsev and Nedospasov, 1960] but has been more vigorously pursued lately in regard to ionospheric disturbances. The linear theory of the mode is reasonably well developed in the short wavelength limit ( $k L \gg 1$ where $k$ is the wave number and $L$ is the scale length of the density gradient). Among the issues considered thus far are the linear properties of the mode in the low-altitude auroral $F$ region [Ossakow and Chaturvedi, 1979; Vickrey et al., 1980], the high-altitude auroral $F$ region [Chaturvedi and Ossakow, 1981], and the auroral $E$ region [Chaturvedi and Ossakow, 1981], as well as studies of the influence of electromagnetic effects [Chaturvedi and Ossakow, 1981], magnetic shear [Huba and Ossakow, 1980], a warm electron beam [Chaturvedi and Ossakow, 1983], velocity shear (P. Satyanarayana and S. L. Ossakow, unpublished manuscript, 1984), and kinetic effects (e.g., finite ion Larmor radius effects, wave-particle resonances; Gary [1984]) on the instability. A nonlinear theory of this instability has also been developed [Chaturvedi and Ossakow, 1979], and numerical simulations of the instability have been performed [Keskinen et al., 1980].

The purpose of this paper is to present analytical and numerical results of the linear properties of the current convective instability in the long-wavelength regime $(k L<1)$. The mathematical analysis is similar to that of the long-wavelength limit of the $\mathbf{E} \times \mathbf{B}$ instability [Huba and Zalesak, 1983]. We

This paper is not subject to U.S. copyright. Published in 1984 by the American Geophysical Union.

Paper number 4A0109. derive a relatively simple dispersion equation of the mode and present simple dispersion relations in both the ion collisional and ion inertial regimes. These analytical results are compared to exact numerical results.

The organization of the paper is as follows. In the next section we derive the differential equation describing the mode. In section 3 we present both analytical and numerical results. Finally, in section 4 we summarize our findings and discuss applications to the auroral ionosphere.

\section{Derivation of Mode Equation}

The plasma configuration and slab geometry used in the analysis are shown in Figure $1 a$. The ambient magnetic field is constant and in the $z$ direction $\left(B=B_{0} \hat{e}_{z}\right)$, the ambient current is constant and in the $z$ direction $\left(J=J_{0} \hat{e}_{z}\right)$, and the density is inhomogeneous in the $x$ direction $\left(n=n_{0}(x)\right.$ ). A weakly collisional plasma is assumed such that $v_{e i} / \Omega_{e} \ll 1, v_{e n} / \Omega_{e} \ll 1$, $v_{i e} / \Omega_{i} \ll 1$, and $v_{i n} / \Omega_{i} \ll 1$ ( $F$ region approximation), where $\Omega_{\alpha}=e B_{0} / m_{\alpha} c$ is the cylotron frequency of species $\alpha, v_{e i}$ refers to electron-ion collisions, $v_{e n}$ to electron-neutral collisions, $v_{i e}$ to ion-electron collisions, and $v_{\text {in }}$ to ion-neutral collisions. Furthermore, we assume that $v_{e n} / \boldsymbol{\Omega}_{e} \ll v_{i n} / \boldsymbol{\Omega}_{i}$ in our analysis. Perturbed quantities are assumed to vary as $\delta p=\delta p(x) \exp \left[i\left(k_{y} y\right.\right.$ $\left.\left.+k_{z} z-\omega t\right)\right]$, and it is assumed that $\omega / \Omega_{i} \ll 1, k \rho_{i} \ll 1$, and $k_{z} \ll k_{y}$, where $\rho_{i}$ is the mean ion Larmor radius. That is, we consider low-frequency, long-wavelength, field-aligned perturbations. We also assume $k_{z} \lambda_{M F P} \ll 1$, where $\lambda_{M F P}$ is the electron mean free path. We neglect temperature effects. Finally, we consider only electrostatic oscillations and assume quasineutrality $\left(n_{e} \simeq n_{i}\right)$.

The fundamental equations used in the analysis are continuity, momentum transfer, and charge conservation in the neutral frame of reference:

$$
\begin{gathered}
\left(\partial n_{z} / \partial t\right)+\nabla \cdot\left(n \mathbf{V}_{\alpha}\right)=0 \\
0=-\frac{e}{m_{e}}\left(\mathbf{E}+\frac{1}{c} \mathbf{V}_{e} \times \mathbf{B}\right)-v_{e n} \mathbf{V}_{e}-v_{e i}\left(\mathbf{V}_{e}-\mathbf{V}_{i}\right) \\
\frac{d \mathbf{V}_{i}}{d t}=\frac{e}{m_{i}}\left(\mathbf{E}+\frac{1}{c} \mathbf{V}_{i} \times \mathbf{B}\right)-v_{i n} \mathbf{V}_{i}-v_{i e}\left(\mathbf{V}_{i}-\mathbf{V}_{e}\right) \\
\nabla \cdot \mathbf{J}=\nabla \cdot\left[n_{e}\left(\mathbf{V}_{i}-\mathbf{V}_{e}\right)\right]=0
\end{gathered}
$$

where $\alpha$ denotes species ( $e$, electrons; $i$, ions), and other variables have their usual meaning. Note that we have neglected electron inertia effects in (2) but have retained ion inertia ef- 


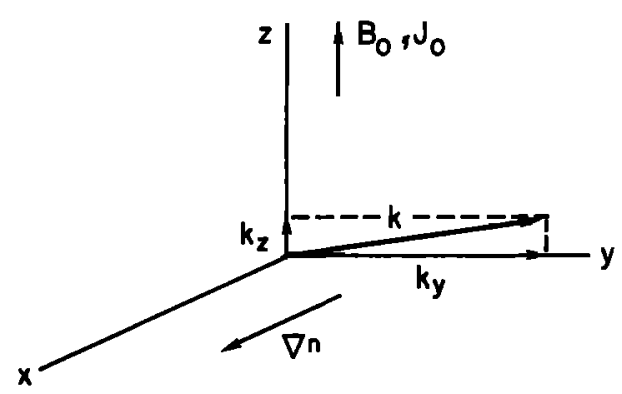

(a)

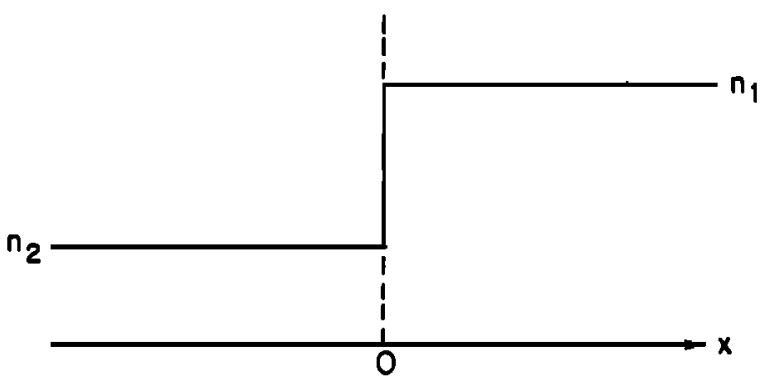

(b)

Fig. 1. Plasma geometry and slab configuration used in the analysis: $(a)$ standard plasma configuration; (b) plasma configuration with a discontinuity in the density of $x=0$.

fects in (3). The equilibrium drifts are given by

$$
\begin{gathered}
\mathbf{V}_{e 0}=0 \\
\mathbf{V}_{i 0}=V_{d} \hat{e}_{z}
\end{gathered}
$$

where we have chosen to work in the electron frame of reference in the $z$ direction. Thus the current is given by $\mathbf{J}=$ en $V_{d} \hat{e}_{z}$.

We now linearize (1)-(3) and take $n=n_{0}+\delta n, V_{a}=V_{\alpha 0}$ $+\delta \mathbf{V}_{a}$, and $\mathbf{E}=-\nabla \phi$, where $\phi$ is the perturbed electrostatic potential. Using (2) and (3), we find that

$$
\begin{gathered}
\delta \mathbf{V}_{e}=-\frac{c}{B} \nabla_{\perp} \phi x \hat{e}_{z}+\frac{\Omega_{e}}{v_{e l}} \frac{c}{B} \nabla_{\|} \phi \hat{e}_{z} \\
\delta \mathbf{V}_{i}=-\frac{c}{B} \nabla_{\perp} \phi x \hat{e}_{z}+i \frac{\tilde{\omega}}{\Omega_{i}} \nabla_{\perp} \phi
\end{gathered}
$$

which can be written as

$$
\begin{gathered}
\delta \mathbf{V}_{e}=-i \frac{c}{B} k_{y} \phi \hat{e}_{x}+\frac{c}{B} \phi^{\prime} \hat{e}_{y}+i \frac{c}{B} \frac{\Omega_{e}}{v_{e i}} k_{z} \phi \hat{e}_{z} \\
\delta \mathbf{V}_{i}=\frac{c}{B}\left[-i k_{y} \phi+i \frac{\tilde{\omega}}{\Omega_{i}} \phi^{\prime}\right] \hat{e}_{x}+\frac{c}{B}\left[-k_{y} \frac{\tilde{\omega}}{\Omega_{i}} \phi+\phi^{\prime}\right] \hat{e}_{y}
\end{gathered}
$$

where $\tilde{\sigma}=\omega-k_{z} V_{d}+i v_{i n}$, and the prime indicates a derivative with respect to $x$. We have neglected collisional effects on the electron motion perpendicular to $B$ and on the ion motion parallel to B. This is justified, since we have taken $v_{e n} / \Omega_{e} \ll$ $\tilde{\omega} / \Omega_{i}$, which is appropriate for auroral ionospheric conditions in the $F$ region.
We now substitute (9) and (10) into (4) and obtain

$$
\left(n_{0} \phi^{\prime}\right)^{\prime}-n_{0} k_{y}^{2}\left(1+i \frac{k_{z}^{2}}{k_{y}^{2}} \frac{\Omega_{i} \Omega_{e}}{\tilde{\omega} v_{e i}}\right) \phi+\frac{B}{c} \frac{\Omega_{i}}{\tilde{\omega}} k_{z} V_{d} \delta n=0
$$

We relate $\delta n$ and $\phi$ by using the electron continuity equation and find that (from (1) and (9))

$$
\delta n=\frac{c}{B} \frac{k_{y}}{\omega}\left[n_{0} \phi^{\prime}-\left(n_{0} \phi\right)^{\prime}+i n_{0} \frac{k_{z}}{k_{y}} \frac{\Omega_{e}}{v_{e i}} k_{z} \phi\right]
$$

Finally, substituting (12) into (11), we arrive at the mode equation for the current convective instability

$$
\begin{aligned}
\left(n_{0} \phi^{\prime}\right)^{\prime}-n_{0} k_{y}{ }^{2}\left[1+i \frac{k_{z}{ }^{2}}{k_{y}{ }^{2}} \frac{\Omega_{l} \Omega_{e}}{\tilde{\omega} v_{e i}}\right. & \left.\left(1-\frac{k_{z} V_{d}}{\omega}\right)\right] \phi \\
& -\frac{k_{z} V_{d}}{\omega} \frac{\Omega_{i}}{\tilde{\omega}} k_{y} n_{o}{ }^{\prime} \phi=0
\end{aligned}
$$

\section{ANALysis of Mode Equation}

The bulk of linear analyses of the current convective instability has made use of the local approximation. That is, it is assumed that $k_{y}{ }^{2} L^{2} \gg k_{x}{ }^{2} L^{2} \gg 1$, where $L=\left(\partial \ln n_{0} / \partial x\right)^{-1}$ is the density gradient scale length and $k_{x}=\partial / \partial x$. With this assumption, one can neglect the first term on the left of (13) and obtain a relatively simple dispersion equation

$$
1+i \frac{k_{z}{ }^{2}}{k_{y}{ }^{2}} \frac{\Omega_{i} \Omega_{e}}{\tilde{\omega} v_{e t}}\left(1-\frac{k_{z} V_{d}}{\omega}\right)-\frac{k_{z}}{k_{y}} \frac{\Omega_{i}}{\tilde{\omega}} \frac{V_{d}}{\omega} \frac{n_{0}{ }^{\prime}}{n_{0}}=0
$$

which has been thoroughly analyzed [Chaturvedi and Ossakow, 1981]. The heart of the local approximation is that the wavelengths of the perturbations are much smaller than the scale length of the density gradient. In this paper we solve (13) in the opposite limit, i.e., the wavelengths of the perturbations are much larger than the scale length of the density gradient $(k L \ll 1)$.

We consider a density profile with a single discontinuity at $x=0$ (see Figure $1 b$ ) given by

$$
n_{0}(x)= \begin{cases}n_{1} & x>0 \\ n_{2} & x<0\end{cases}
$$

For $x \neq 0$ and $n_{0}{ }^{\prime}=0$, (13) reduces to

$$
\phi^{\prime \prime}-k_{y}{ }^{2} \Gamma^{2} \phi=0
$$

where

$$
\Gamma^{2}=1+i \frac{k_{z}^{2}}{k_{y}^{2}} \frac{\Omega_{i} \Omega_{e}}{\tilde{\omega} v_{e i}}\left(1-\frac{k_{z} V_{d}}{\omega}\right)
$$

The solution to (16) is taken to be

$$
\phi(x)=\phi_{1} e^{-k_{y} \Gamma x}+\phi_{2} e^{k_{y} \Gamma x}
$$

Since the modes are assumed to be bounded as $x \rightarrow \pm \infty$, we note that

$$
\phi(x)= \begin{cases}\phi_{1} e^{-k_{y} \Gamma x} & x>0 \\ \phi_{2} e^{k_{y} \Gamma x} & x<0\end{cases}
$$

where it is assumed that $\omega \gg k_{z} V_{d}$ (to be shown a posteriori).

We require that the tangential component of the electric field be continuous at $x=0$ [Hasegawa, 1971], which means that $\phi$ is continuous at $x=0$. This is equivalent to requiring that the interface velocity and the fluid velocity perpendicular 
to the interface be equal [Chandrasekhar, 1961], i.e., $\delta V_{x}$ is continuous at the discontinuity. Thus $\phi_{1}=\phi_{2}$ in (19), so that

$$
\phi(x)= \begin{cases}\phi_{0} e^{-k_{y} \Gamma x} & x>0 \\ \phi_{0} e^{k_{y} \Gamma x} & x<0\end{cases}
$$

To obtain the dispersion equation, we integrate (13) across the discontinuity at $x=0$. Thus we have

$$
\int_{-\varepsilon}^{\varepsilon}\left(n_{0} \phi^{\prime}\right)^{\prime} d x=\int_{-\varepsilon}^{\varepsilon}\left[n_{0} k_{y}{ }^{2} \Gamma^{2} \phi-\frac{k_{z} V_{d}}{\omega} \frac{\Omega_{i}}{\tilde{\omega}} k_{y} n_{0}{ }^{\prime} \phi\right] d x
$$

Since $\phi$ is continuous across the boundary at $x=0$, it is found that (21) leads to

$$
\left(n_{0} \phi^{\prime}\right)_{1}-\left(n_{0} \phi^{\prime}\right)_{2}=-\frac{k_{z} V_{d}}{\omega} \frac{\Omega_{i}}{\tilde{\omega}} k_{y}\left(n_{1}-n_{2}\right) \phi_{0}
$$

where $(1,2)$ indicate the region $x>0(+\varepsilon)$ and $x<0(-\varepsilon)$, respectively. Substituting (20) into (22), and letting $\varepsilon \rightarrow 0$, we find that

$$
\omega \tilde{\omega} \Gamma=k_{2} V_{d} \Omega_{i} \frac{n_{1}-n_{2}}{n_{1}+n_{2}}
$$

Equation (23) is the dispersion equation that describes the long-wavelength modes of the current convective instability. From (23) we note that $\omega / k_{z} V_{d} \sim \Omega_{i} / \tilde{\omega} \gg 1$, so that we can take

$$
\Gamma^{2}=1+i \frac{k_{z}^{2}}{k_{y}^{2}} \frac{\Omega_{i}}{\tilde{\omega}} \frac{\Omega_{e}}{v_{e i}}
$$

and $\tilde{\omega} \simeq \omega+i v_{i n}$.

\subsection{Collisional Limit}

We consider the collisional limit $v_{i n} \gg \omega$ so that $\tilde{\omega} \simeq i v_{\text {in }}$ in (23) and (24). We find that

$$
\gamma_{n l}=-k_{z} V_{d} \frac{\Omega_{i}}{v_{i n}} \frac{1}{\Gamma} \frac{n_{1}-n_{2}}{n_{1}+n_{2}}
$$

with

$$
\Gamma=\left(1+\frac{k_{z}^{2}}{k_{y}^{2}} \frac{\Omega_{i}}{v_{i n}} \frac{\Omega_{e}}{v_{e i}}\right)^{1 / 2}
$$

where the subscript $n l$ denotes nonlocal. Instability can occur for $k_{z} V_{d}\left(n_{1}-n_{2}\right) /\left(n_{1}+n_{2}\right)<0$.

It is interesting to compare the growth rate of the instability in the long-wavelength limit ( $k L \ll 1$, i.e., nonlocal) to that of the short-wavelength limit ( $k L \gg 1$, i.e., local). Assuming $v_{i n} \gg$ $\omega$, we find from (14) that the short-wavelength growth rate (local growth rate $\gamma_{l}$ ) is given by

$$
\gamma_{l}=-\frac{k_{z}}{k_{y}} \frac{\Omega_{i}}{v_{i n}} \frac{V_{d}}{L}\left(1+\frac{k_{z}^{2}}{k_{y}^{2}} \frac{\Omega_{e}}{v_{i n}} \frac{\Omega_{e}}{v_{e i}}\right)^{-1}
$$

where $L=\left(n_{0}^{\prime} / n_{0}\right)_{\max }{ }^{-1}$. Defining an effective wave number $\hat{k}=\left(k_{y}{ }^{2}+k_{z}{ }^{2}\left(\Omega_{e} \Omega_{i} / v_{i n} v_{e i}\right)\right)^{1 / 2}$, we can rewrite (25) in terms of (27), i.e.,

$$
\gamma_{n l}=k L \frac{n_{1}-n_{2}}{n_{1}+n_{2}} \gamma_{l}
$$

For $n_{1} \gg n_{2}$ we have the simple relation $\gamma_{n l} \simeq k L \gamma_{l}$ so that (1) $\gamma_{n l}$ is proportional to the "wavenumber" $\hat{k}$ and (2) $\gamma_{n l} \ll \gamma_{l}$ since we have assumed $k L \ll 1$.

We now compare the analytical expressions derived for the growth rates ((25) and (27)) with the numerical solution of (13). We choose a density profile given by

$$
n(x)=n_{0} \frac{1+\varepsilon \tanh (x / a)}{1-\varepsilon}
$$

so that

$$
\frac{n^{\prime}}{n}=\frac{1}{a} \frac{\varepsilon \operatorname{sech}^{2}(x / a)}{1+\varepsilon \tanh (x / a)}
$$

We take $\varepsilon=0.8$ so that $n^{\prime} / n$ is a maximum at $x / a=-0.55$. We find then that $\left(n^{\prime} / n\right)_{\max } \simeq(1 / a)$ so that $L \simeq a$ in (27)-(30).

We consider the following physical parameters for the comparison of analytical and numerical results: $v_{e i} / \Omega_{e}=10^{-4}$,

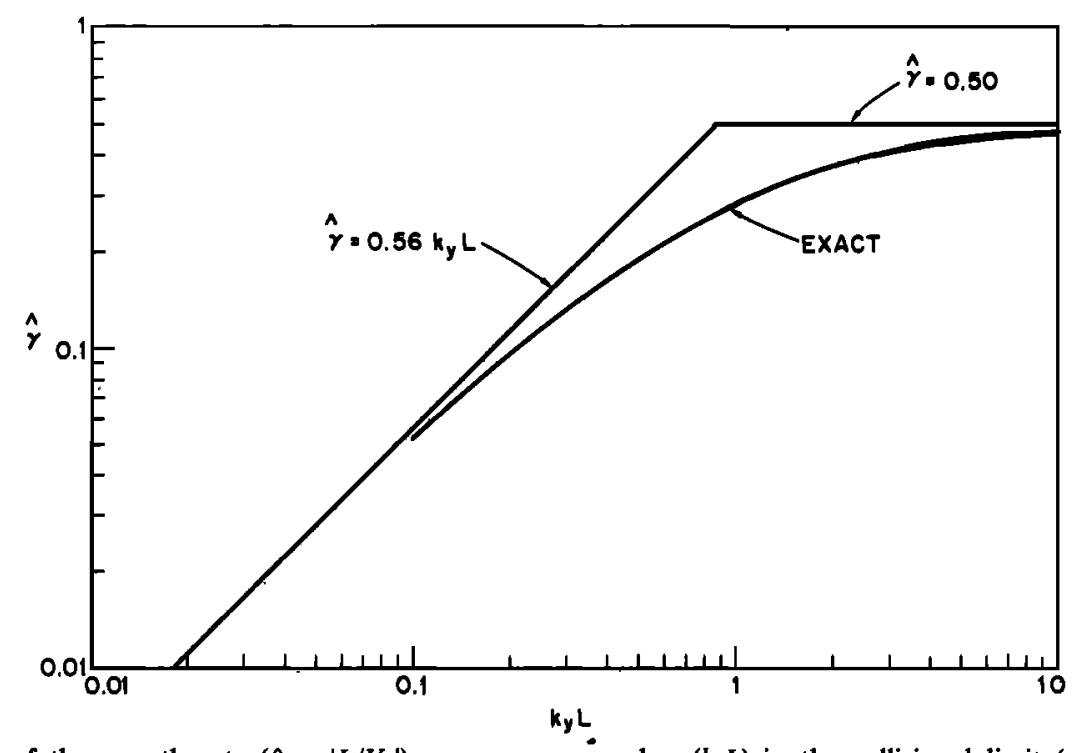

Fig. 2. Plot of the growth rate $\left(\hat{y}=\gamma \mid L / V_{d}\right)$ versus wave number $\left(k_{y} L\right)$ in the collisional limit $\left(v_{\text {in }} \gg \omega\right)$ for both analytical and numerical results. The parameters used are $v_{e i} / \Omega_{e}=10^{-4}, v_{i n} / \Omega_{i}=10^{-4}$, and $k_{z} / k_{y}=10^{-4}$. The analytical results are based upon (31) and labeled accordingly. The numerical results are based upon (13) and (29) with $\varepsilon=0.8$. 
$v_{i n} / \Omega_{i}=10^{-4}$, and $k_{z} / k_{y}=10^{-4}$ [Rino et al., 1978; Ossakow and Chaturvedi, 1979]. We also consider the normalization $\hat{\omega} \equiv \omega\left|L / V_{d}\right|$, where it is assumed that $L / V_{d}<0$ so that $\gamma>0$. We find then that the analytical expression for the growth rate is

$$
\hat{\gamma}= \begin{cases}0.50 & k L \gg 1 \\ 0.56 k_{y} L & k L \ll 1\end{cases}
$$

We solve (13) numerically for the density profile given by (29) and the parameters described above. In Figure 2 we plot $\hat{\gamma}$ versus $k_{y} L$. The numerical solution is plotted in the regime $0.1<k_{y} L<10.0$ and is labeled "exact." As is evident, the numerical solution asymptotes to the analytical expression (31) in both the short-wavelength $(k L \gg 1)$ and long-wavelength $(k \ll 1)$ limits. Thus (25) and (27) provide good estimates of the current convective instability in the long-wavelength and short-wavelength limits, respectively.

\subsection{Inertial Limit}

We now consider the ion inertial limit given by $\omega \gg v_{\text {in }}$ so that $\tilde{\omega} \simeq \omega$ in (23) and (24). The dispersion equation is

$$
\omega^{2}\left(1+i \frac{k_{z}^{2}}{k_{y}^{2}} \frac{\Omega_{i}}{\omega} \frac{\Omega_{e}}{v_{e i}}\right)^{1 / 2}=k_{z} V_{d} \Omega_{i} \frac{n_{1}-n_{2}}{n_{1}+n_{2}}
$$

We obtain a simple expression for the growth rate by assuming that $\omega / \Omega_{l} \ll\left(k_{z}{ }^{2} / k_{y}{ }^{2}\right)\left(\Omega_{e} / v_{e l}\right)$. In this limit, (32) becomes

$$
\omega^{3}=-i k_{y}{ }^{2} V_{d}{ }^{2} \Omega_{i} \frac{v_{e i}}{\Omega_{e}}\left(\frac{n_{1}-n_{2}}{n_{1}+n_{2}}\right)^{2}
$$

Again, we consider the normalization $\hat{\omega}=\omega\left|L / V_{d}\right|$ and take $L / V_{d}<0$. We find that

$$
\hat{\gamma}_{n l}=\left(\frac{v_{e i}}{\Omega_{e}} \frac{\Omega_{i} L}{V_{d}}\right)^{1 / 3}\left(k_{y} L\right)^{2 / 3}\left(\frac{n_{1}-n_{2}}{n_{1}+n_{2}}\right)^{2 / 3}
$$

In this limit it is interesting to note that $\hat{\gamma}$ scales as $\left(k_{y} L\right)^{2 / 3}$ and is independent of $k_{z} / k_{y}$.

We contrast (34) to the growth rate in the short-wavelength limit $(k L \gg 1)$. From (14) we find that

$$
\hat{\gamma}_{l}=-\frac{1}{2} \frac{k_{z}^{2}}{k_{y}^{2}} \frac{\Omega_{e}}{v_{e i}}\left|\frac{L \Omega_{i}}{\Omega_{d}}\right|\left[1-\left(1+4\left|\frac{V_{d}}{L \Omega_{i}}\right| \frac{k_{y}^{3}}{k_{z}{ }^{3}} \frac{v_{e i}{ }^{2}}{\Omega_{e}{ }^{2}}\right)^{1 / 2}\right]
$$

Assuming that $4\left|V_{d} / L \Omega_{i}\right|\left(k_{y}{ }^{3} / k_{z}{ }^{3}\right)\left(v_{e i}{ }^{2} / \Omega_{e}{ }^{2}\right) \ll 1$, we find that (36) reduces to

$$
\hat{\gamma}_{t}=\frac{k_{y}}{k_{z}} \frac{v_{e i}}{\Omega_{e}}
$$

Following Chaturvedi and Ossakow [1981], we maximize $\hat{\gamma}_{t}$ with respect to $k_{z} / k_{y}$ and find that the maximum local growth rate $\left(\hat{\gamma}_{l m}\right)$ in the inertial limit is

$$
\hat{\gamma}_{l m}=\left(\frac{v_{e i}}{\Omega_{e}} \frac{L \Omega_{i}}{4 V_{d}}\right)^{1 / 3}
$$

We can express the long-wavelength growth rate in terms of the maximum short-wavelength growth rate to obtain

$$
\hat{\gamma}_{n l} \simeq \alpha \gamma_{l m}\left[k_{y} L \frac{n_{1}-n_{2}}{n_{1}+n_{2}}\right]^{2 / 3}
$$

where $\alpha$ is a numerical factor of order unity. Thus, for $n_{1} \gg n_{2}$, we find that $\gamma_{n l} \simeq\left(k_{y} L\right)^{2 / 3} \gamma_{l m}$ in contrast to the collisional limit in which $\hat{\gamma}_{n l}$ scales as $k_{y} L$.

We now compare the analytical expressions for the growth rate with numerical results based upon (13). We choose the same density profile as in the collisional case ((29) with $\varepsilon=0.8$ ) and take $v_{e i} / \Omega_{e}=10^{-4}, k_{z} / k_{y}=10^{-4}, v_{i n} / \Omega_{i}=0$, and $\left|L \Omega_{i} / V_{d}\right|=10^{4}$. For these parameters the assumption that led to (36) is not satisfied, and the analytical expression for $\hat{\gamma}_{l}$, given by (35), must be used. The analytical growth rate is given by

$$
\hat{\gamma}= \begin{cases}0.62 & k_{y} L \gg 1 \\ 0.86\left(k_{y} L\right)^{2 / 3} & k_{y} L \ll 1\end{cases}
$$

The results of the comparison are shown in Figure 3, where

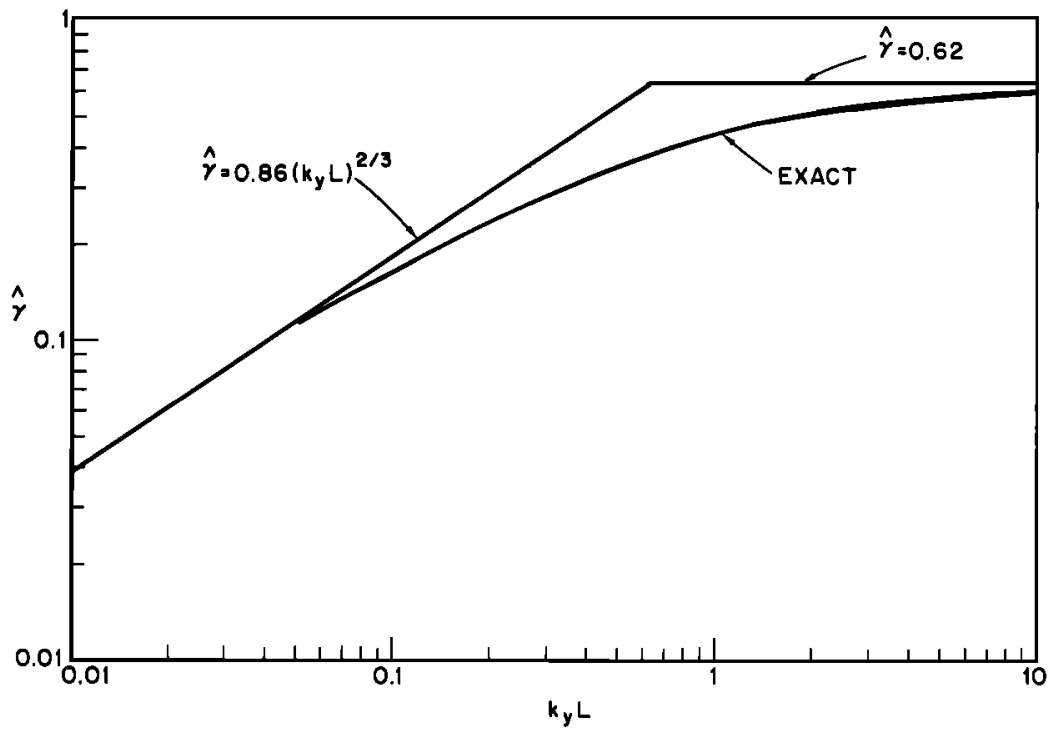

Fig. 3. Plot of the growth rate $\left(\hat{\gamma}=\gamma \mid L / V_{\mathrm{d}}\right)$ versus wave number $\left(k_{y} L\right)$ in the inertial limit $\left(v_{i n} \ll \omega\right)$ for both analytical and numerical results. The parameters used are $v_{e i} / \Omega_{e}=10^{-4}, k_{z} / k_{y}=10^{-4}$, and $v_{i n} / \Omega_{i}=0$. The analytical results are based upon (39) and labeled accordingly. The numerical results are based upon (13) and (29) with $\varepsilon=0.8$. 
we plot $\hat{\gamma}$ versus $k_{y} L$. The growth rate $\hat{\gamma}$ given by (39) is plotted as shown on Figure 3, and the numerical results are labeled "exact." Again, excellent agreement is found between the analytical and numerical results in both the short-wavelength and long-wavelength limits.

\section{Discussion}

We have presented an analysis of the current convective instability in the long-wavelength limit $(k L \ll 1)$. The principal result of the paper is the derivation of a relatively simple dispersion equation that describes the instability in the longwavelength limit ((22)). This equation is solved analytically in two limits: collisional $\left(v_{i n} \gg \omega\right)$ and inertial $\left(v_{i n} \ll \omega\right)$. We find that in the collisional limit the growth rate scales as $k$, while in the inertial limit it scales as $k^{2 / 3}$. We have also presented a comparison of the analytical results with numerical results and have found very good agreement.

We now discuss the application of these results to the auroral ionosphere. Chaturvedi and Ossakow [1981] have discussed the relevance of the short-wavelength (or local) current convective instability to both the low-altitude $(\sim 400 \mathrm{~km})$ auroral $F$ region (collisional limit) and to the high-altitude $(\sim 1000$ $\mathrm{km}$ ) auroral $F$ region (inertial limit). For the low-altitude $F$ region they find that $\gamma_{l} \sim 3 \times 10^{-3} \mathrm{~s}^{-1}$ for $V_{d} \sim 500 \mathrm{~m} / \mathrm{s}$, $v_{e i} \sim 5 \times 10^{2} \mathrm{~s}^{-1}, \quad v_{i n} \sim 5 \times 10^{-2} \mathrm{~s}^{-1}, L \sim 50 \mathrm{~km}$, and $m_{e} / m_{i} \sim 3 \times 10^{-5}$; for the high-altitude $F$ region they also find that $\gamma_{1} \sim 3 \times 10^{-3} \mathrm{~s}^{-1}$, but for $v_{e i} \sim 30 \mathrm{~s}^{-1}, v_{i n} \leqslant 10^{-3} \mathrm{~s}^{-1}$, and other parameters it is the same as the low-altitude $F$ region. From our analysis we would predict that the longwavelength modes $(k L<1)$ would have much longer growth times $(1 / \gamma)$, particularly in the low-altitude $F$ region.

There is a difficulty in applying the long-wavelength results to the auroral $F$ region for large density scale lengths $(L \sim 50$ $\mathrm{km}$ ). The perpendicular wavelengths associated with the longwavelength modes are such that $k_{\perp} L<1$, which leads to $\lambda_{\perp}>$ $2 \pi L \sim 300 \mathrm{~km}$. However, the perpendicular spatial scale size of observed auroral $F$ region density enhancements, which can provide the zero-order density gradients to drive the instability, can be of this magnitude (few hundreds of kilometers) [Vickrey et al., 1980; R. T. Tsunoda and J. F. Vickrey, unpublished manuscript, 1983] so that it is difficult to satisfy the condition $k_{\perp} L<1$. On the other hand, much smaller scale sizes of auroral structure occur during discrete aurora. Discrete aurora appear to have two distinct scale sizes. One is tens of kilometers and is associated with inverted $V$ precipitation. The other is of the order of a kilometer and is associated with discrete auroral arc elements [Davis, 1978]. The auroral arc elements appear to be imbedded in the larger inverted $V$ structure. Thus application of our theory to structure in discrete auroral arc elements leads to perpendicular wavelengths $\lambda_{\perp} \gtrsim$ few kilometers, which can satisfy the requirement $k_{\perp} L<1$.

Finally, several aspects of the present theory of the current convective instability deserve comment. First, the parallel wavelengths of the irregularities are much larger than the perpendicular wavelengths. For typical parameters we find that $k_{||} / k_{\perp} \sim 10^{-4}$ so that $\lambda_{||} \sim 10^{4} \lambda_{\perp}$. The instability in the longwavelength limit can then produce parallel wave structures $\lambda_{\|} \gtrsim 10^{4} \mathrm{~km}$, which is larger than the parallel system size. Thus it is important to develop an appropriate theory to consider the finite parallel extent of the auroral ionosphere (e.g., J. L. Sperling, unpublished manuscript, 1983). Second, electro- magnetic effects also need to be considered in a more detailed theory. Chaturvedi and Ossakow [1981] investigated these effects on the local theory of the current convective instability and found them to be negligible. However, the electromagnetic corrections are proportional to $\omega_{p e}^{2} / c^{2} k^{2}$ and may become large in the long-wavelength limit since $k L<1$. We are presently investigating both of the above mentioned eflects.

Acknowledgments. We thank Mike Keskinen for helpful conversations and for a critical reading of the manuscript. This research has been supported by the Defense Nuclear Agency and the Office of Naval Research.

The Editor thanks S. P. Gary and J. L. Sperling for their assistance in evaluating this paper.

\section{REFERENCES}

Chandrasekhar, S., Hydrodynamic and Hydromagnetic Stability, Oxford University Press, New York, 1961.

Chaturvedi, P. K., and S. L. Ossakow, Nonlinear stabilization of the current convective instability in the diffuse aurora, Geophys. Res. Lett., 6, 957, 1979.

Chaturvedi, P. K., and S. L. Ossakow, The current instability as applied to the auroral ionosphere, J. Geophys. Res., 86, 4811, 1981.

Chaturvedi, P. K., and S. L. Ossakow, Effect of an electron beam on the current convective instability, J. Geophys. Res., 88, 4114, 1983.

Davis, T. N., Observed characteristics of auroral forms, Space Sci. Rev., 22, 77, 1978.

Fremouw, E. J., C. L. Rino, R. C. Livingston, and M. C. Cousins, A persistent subauroral scintillation enhancement observed in Alaska, Geophys. Res. Lett., 4, 539, 1977.

Fremouw, E. J., et al., The HILAT program, Eos Trans. AGU, 64, $163,1983$.

Gary, S. P., Kinetic theory of current and density drift instabilities with weak charged-neutral collisions, J. Geophys. Res., 89, 179, 1984.

Hoh, F. C., and B. Lehnert, Diffusion processes in a plasma column in a longitudinal magnetic field, Phys. Fluids, 3, 600, 1960.

Huba, J. D., and S. L. Ossakow, Influence of magnetic shear on the current convective instability in the diffuse aurora, J. Geophys. Res., $85,6874,1980$.

Huba, J. D., and S. T. Zalesak, Long wavelength limit of the $E \times B$ instability, J. Geophys. Res., 88, 10,263, 1983.

Kadomtsev, B. B., and A. V. Nedospasov, Instability of the positive column in a magnetic field and the "anomalous diffusion effect," $J$. Nucl. Energy, Part C, 1, 230, 1960.

Keskinen, M. J., and S. L. Ossakow, Nonlinear evolution of plasma enhancements in the diffuse auroral $F$ region ionosphere, 1, Longwavelength irregularities, J. Geophys. Res., 87, 144, 1982.

Keskinen, M. J., and S. L. Ossakow, Theories of high-latitude ionospheric irregularities: A review, Radio Sci., 18, 1077, 1983.

Keskinen, M. J., S. L. Ossakow, and B. E. McDonald, Nonlinear evolution of diffuse auroral $F$ region ionospheric irregularities, $G e o-$ phys. Res. Lett., 7, 573, 1980.

Lehnert, B., Diffusion processes in the positive column in a longitudinal magnetic field, in Proc. 2nd Geneva Conf. Peaceful Uses At. Energy, 32, 349, 1958.

Ossakow, S. L., and P. K. Chaturvedi, Current convective instability in the diffuse aurora, Geophys. Res. Lett., 6, 332, 1979.

Rino, C. L., R. C. Livingston, and S. J. Matthews, Evidence for sheetlike auroral ionospheric irregularities, Geophys. Res. Lett., 5, 1039, 1978.

Vickrey, J. F., C. L. Rino, and T. A. Potemra, Chatanika/Triad observations of unstable ionization enhancements in the auroral $F$ region, Geophys. Res. Lett., 7, 789, 1980.

J. D. Huba, Geophysical and Plasma Dynamics Branch, Plasma Physics Division, Naval Research Laboratory, Washington, D. C. 20375.

(Received November 30, 1983; revised January 16, 1984; accepted January 17,1984 .) 